\title{
Legendre Wavelet and He's Homotopy Perturbation Methods for Linear Fractional Integro-Differential Equations
}

\author{
M. H. Saleh \\ Mathematics Department \\ Faculty of Science \\ Zagazig University, Egypt
}

\author{
A.S. Nagdy \\ Mathematics Department \\ Faculty of Science \\ Zagazig University, Egypt
}

\author{
M. E. M. Alngar \\ Mathematics Department \\ Faculty of Science \\ Zagazig University, Egypt
}

\begin{abstract}
In this paper, the Legendre wavelet method (LWM) and He's Homotopy perturbation method (HPM) are applied to approximate solution for linear fractional integro-differential equation with initial condition. A comparison between these methods takes place. Numerical examples are presented to illustrste the efficiency and accuracy of the proposed methods.
\end{abstract}

\section{General Terms}

Numerical solutions, Fractional integro-differential equations.

\section{Keywords}

Fractional integro-differential equations, Legendre wavelet method, He's homotopy perturbation method, Caputo fractional derivative, Riemann-Liouville.

\section{INTRODUCTION}

In recent years, for solving linear and nonlinear mathematical, engineering and physical problems, many of the numerical methods are used for seeking approximate solutions such as Collocation method, Taylor expansion method, Adomian decomposition method, Legendre wavelet method and Homotopy perturbation method, see $([3,4,7,12,13])$. The analytic results on existence and uniqueness of problems solutions to fractional differential equations have been investigated by many authors $[1,6,11,14]$. In this paper will be taken the fractional integro-differential equations with a Caputo fractional derivative of the type

$D_{*}^{\alpha} y(t)=f(t)+P(t) y(t)+\int_{0}^{t} k(t, s) y(s) d s, \mathrm{t}$

$\in[0,1]$

with the initial condition

$y(0)=\beta$,

$0<\alpha \leq 1$

where $D_{*}^{\alpha}$ is Caputo's fractional derivative and $\alpha$ is a parameter describing the order of the fractional derivative, $\beta$ is real constant, $\mathrm{f}(\mathrm{t})$ and $k(t, s)$ are given that can be approximated by Taylor polynomials. We applied the LWM and HPM for approximating the solution of linear fractional integro-differential equation. The outline of this paper is as follows: In section 2, we present some definitions. Sections 3, contains the application of Legendre wavelet method. Section 4, contains the application of He's homotopy perturbation method. Finally, sec.5 devoted to illustrste some numerical examples on mentioned methods.

\section{SOME DEFINITIONS AND NOTATIONS}

Definition 2.1. A real function $f(x), x>0$, is said to be in the space $C_{\alpha}, \alpha \in \mathbb{R}$, if there exists a real number $p>\alpha$, such that $f(x)=x^{p} f_{1}(x)$, where $f_{1}(x) \in C[0, \infty)$.

Definition 2.2. A real function $f(x), x>0$, is said to be in the space $C_{\alpha}^{k}, k \in \mathbb{N} \cup\{0\}$, if $f^{k} \in C_{\alpha}$.

Definition 2.3. [6] $D^{\alpha}$ ( $\alpha$ is real) denotes the fractional differential operator of order $\alpha$ in the sense of RiemannLiouville, defined by:

$$
\begin{aligned}
& D^{\alpha} f(x) \\
& \frac{1}{\Gamma(n-\alpha)} \frac{d^{n}}{d x^{n}} \int_{0}^{x} \frac{f(t)}{(x-t)^{\alpha-n+1}} d t, 0 \leq n-1<\alpha \leq n, \\
& \frac{d^{n} f(x)}{d x^{n}}, \\
& \alpha=n \in \mathbb{N} .
\end{aligned}
$$

Definition 2.4. [6] $I^{\alpha}$ denotes the fractional integral operator of order $\alpha$ in the sense of Riemann-Liouville, defined by:

$I^{\alpha} f(x)=D^{-\alpha} f(x)$
$= \begin{cases}\frac{1}{\Gamma(\alpha)} \int_{0}^{x} \frac{f(t)}{(x-t)^{1-\alpha}} d t, \alpha>0, x>0, \\ f(x), & \alpha=0 .\end{cases}$

Some basic properties of fractional integral and differential operator are listed below [6]: for $f \in C_{\mu}, \mu \geq-1, \gamma \geq-1$, $\alpha, \beta \geq 0$ :

1. $I^{\alpha} I^{\beta} f(x)=I^{\alpha+\beta} f(x)=I^{\beta} I^{\alpha} f(x)$.

2. $I^{\alpha} x^{\gamma}=\frac{\Gamma(\gamma+1)}{\Gamma(\alpha+\gamma+1)} x^{\alpha+\gamma}$.

3. $I^{\alpha} \in C_{0}$.

4. $D^{\alpha} D^{\beta} f(x)=D^{\alpha+\beta} f(x)$.

5. $D^{\alpha} I^{\alpha} f(x)=f(x)$.

6. $I^{\alpha} D^{\alpha} f(x)=f(x)-\sum_{k=0}^{m-1} f^{k}\left(0^{+}\right) \frac{x^{k}}{k !}, m-1<\alpha \leq$ $m \in \mathbb{N}$.

7. $D^{\alpha} x^{\gamma}=\frac{\Gamma(\gamma+1)}{\Gamma(\gamma+1-\alpha)} x^{\gamma-\alpha}, x>0$. 
Definition 2.5. [6] Let $f \in C_{-1}^{n}, n \in \mathbb{N}$. Then the Caputo fractional derivative of $f(x)$, defined by

$$
\begin{aligned}
& D_{*}^{\alpha} f(x) \\
& \frac{1}{\Gamma(n-\alpha)} \int_{0}^{x} \frac{f^{(n)}(t)}{(x-t)^{\alpha-n+1}} d t, 0 \leq n-1<\alpha \leq n, \\
& \frac{d^{n} f(x)}{d x^{n}}, \quad \alpha=n \in \mathbb{N} .
\end{aligned}
$$

To obtain a numerical schema for the approximation of Caputo derivative, we can use a representation that has been introduced by Elliots [2],

$D_{*}^{\alpha} f(x)=\frac{1}{\Gamma(-\alpha)} \int_{0}^{x} \frac{f(t)-f(0)}{(x-t)^{1+\alpha}} d t$,

where the integral in equation (2.4) is a Hadamard finite-part integral.

Definition 2.6. The following functions

$\psi_{k, n}(t)=\left|a_{0}\right|^{\frac{k}{2}} \psi\left(a_{0}^{k} t-n b_{0}\right)$,

form a family of discrete wavelets, where $a_{0}>1, b_{0}>0$ and $\mathrm{n}, \mathrm{k}$ are positive integer and $\psi$ is given function called mother wavelet. Moreover, the functions

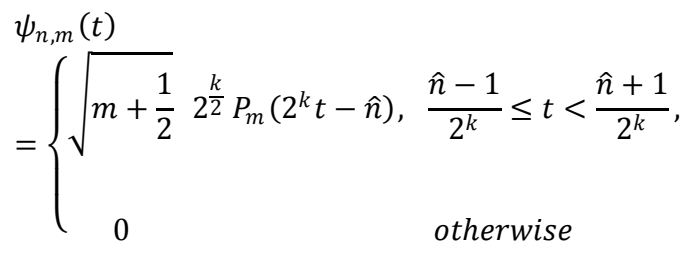

where

$P_{m}(t)=\frac{1}{2^{m} m !} \frac{d^{m}}{d t^{m}}\left[\left(t^{2}-1\right)^{m}\right], \quad m=0,1,2, \ldots$

are called Legendre wavelets polynomials, where $\hat{n}=2 n-$ $1, n=1, \ldots \ldots, 2^{k-1}, k \in \mathbb{N}, t \in[0,1]$ and $\mathrm{m}$ is the order of the Legendre polynomial $P_{m}$

\section{LEGENDRE WAVELET METHOD}

Legendre wavelets method, $[4,15]$, are commonly used for the numerical solution of integral equations.

Consider the equation (1.1) with the initial condition (1.2).

The exact solution of (1.1) can be expanded as Legendre wavelets series as:

$\mathrm{y}(\mathrm{t})=\sum_{n=1}^{\infty} \sum_{m=0}^{\infty} C_{n m} \psi_{n, m}(t)$,

where $\psi_{n, m}(t)$ is given by definition (2.5). We approximate the solution $\mathrm{y}(\mathrm{t})$ by the truncated series

$Y_{K, M}(\mathrm{t})=\sum_{n=1}^{2^{K-1}} \sum_{m=0}^{M-1} C_{n m} \psi_{n, m}(t)$

then a total number of $2^{K-1} M$ conditions should exist for determination of $2^{K-1} M$ coefficients

$C_{10}, \ldots, C_{1 M-1}, C_{21}, \ldots, C_{2 M-1}, \ldots, C_{2^{k-1} 0}, \ldots, C_{2^{k-1} M-1}$.

By the initial condition we obtain,

$Y_{K, M}(0)=\sum_{n=1}^{2^{K-1}} \sum_{m=0}^{M-1} C_{n m} \psi_{n, m}(0)=\beta$.
We see that there should be $2^{K-1} M-1$ extra conditions to recover the unknown coefficients $C_{n m}$. These conditions can be obtained by substituting equation (3.1) in equation(1.1),

$$
\begin{aligned}
& \sum_{n=1}^{2^{K-1}} \sum_{m=0}^{M-1} C_{n m} D_{*}^{\alpha} \psi_{n, m}(t) \\
& =f(t)+\sum_{n=1}^{2^{K-1}} \sum_{m=0}^{M-1} C_{n m} P(t) \psi_{n, m}(t) \\
& +\sum_{n=1}^{2^{K-1}} \sum_{m=0}^{M-1} C_{n m} \int_{0}^{t} k(t, s) \psi_{n, m}(s) d s .
\end{aligned}
$$

Now we assume equation (3.3) is exact at $2^{K-1} M-1$ points $x_{i}$ as:

$$
\begin{aligned}
& \sum_{n=1}^{2^{K-1}} \sum_{m=0}^{M-1} C_{n m} D_{*}^{\alpha} \psi_{n, m}\left(x_{i}\right) \\
& =f\left(x_{i}\right)+\sum_{n=1}^{2^{K-1}} \sum_{m=0}^{M-1} C_{n m} P\left(x_{i}\right) \psi_{n, m}\left(x_{i}\right) \\
& +\sum_{n=1}^{2_{i}-1} \sum_{m=0}^{M-1} C_{n m} \int_{0}^{x_{i}} k\left(x_{i}, s\right) \psi_{n, m}(s) d s .
\end{aligned}
$$

The best choice of the $x_{i}$ points are the zeros of the shifted chebyshev of degree $2^{K-1} M-1$ in the interval $[0,1]$ that is

$x_{i}=\frac{s_{i}+1}{2}$, where $s_{i}=\cos \left(\frac{\pi i}{2^{K-1} M-1}\right)$,

Approximating $D_{*}^{\alpha} \psi_{n, m}$ using Diegthelm method [10] on the representation that has been given by equation (2.4), we get

$$
\begin{aligned}
& D_{*}^{\alpha} \psi_{n, m}\left(x_{i}\right) \\
& =\frac{1}{\Gamma(-\alpha)} \int_{0}^{x_{i}} \frac{\psi_{n, m}(s)-\psi_{n, m}(0)}{\left(x_{i}-s\right)^{1+\alpha}} d s \\
& =\frac{x_{i}^{-\alpha}}{\Gamma(-\alpha)} \int_{0}^{1} \frac{\psi_{n, m}\left(x_{i}-x_{i} \omega\right)-\psi_{n, m}(0)}{\omega^{1+\alpha}} d \omega \\
& \simeq \sum_{r=0}^{L} \omega_{r}\left(\psi_{n, m}\left(x_{i}-\frac{x_{i} r}{L}\right)-\psi_{n, m}(0)\right)
\end{aligned}
$$

where $L \in \mathbb{N}$ and the weights $\omega_{r}$ are given by

$$
\begin{aligned}
& \alpha(1-\alpha) L^{-\alpha} \frac{\Gamma(-\alpha)}{x_{i}^{-\alpha}} \omega_{r} \\
& = \begin{cases}-1, & \text { if } r=0, \\
2 r^{1-\alpha}-(r-1)^{1-\alpha}-(r+1)^{1-\alpha}, & \text { if } r=1, \ldots, L-1, \\
(\alpha-1) r^{-\alpha}-(r-1)^{1-\alpha}+r^{1-\alpha}, & \text { if } r=L .\end{cases}
\end{aligned}
$$

Then equation (3.4) becomes

$$
\begin{aligned}
& \sum_{n=1}^{2^{K-1}} \sum_{m=0}^{M-1} \sum_{r=0}^{L} \omega_{r}\left(\psi_{n, m}\left(x_{i}-\frac{x_{i} r}{L}\right)-\psi_{n, m}(0)\right) C_{n m} \\
& =f\left(x_{i}\right)+\sum_{n=1}^{2^{K-1}} \sum_{m=0}^{M-1} C_{n m} P\left(x_{i}\right) \psi_{n, m}\left(x_{i}\right) \\
& +\sum_{n=1}^{2_{i}} \sum_{m=0}^{M-1} C_{n m} \int_{0}^{2_{i}} k\left(x_{i}, s\right) \psi_{n, m}(s) d s .
\end{aligned}
$$

Combine equation (3.2) and equation (3.6) to obtain $2^{K-1} M$ linear equations from which we can compute the unknown coefficients $c_{n m}$. 


\section{HOMOTOPY PERTURBATION METHOD}

To illustrate the basic concepts of HPM for linear fractional Integro-differential equations, consider equation (1.1) with initial conditions (1.2). According to $\operatorname{HPM}[5,7,8$, 9], we construct the following homotopy:

$$
\begin{aligned}
(1-P) D_{*}^{\alpha} y(t)+ & P\left(D_{*}^{\alpha} y(t)-f(t)-P(t) y(t)\right. \\
& \left.-\int_{0}^{t} k(t, s) y(s) d s\right)=0,
\end{aligned}
$$

or

$$
\begin{aligned}
D_{*}^{\alpha} y(t)=P(f(t) & +P(t) y(t) \\
& \left.\left.+\int_{0}^{t} k(t, s) y(s) d s\right)\right),
\end{aligned}
$$

where $P \in[0,1]$ is an embedding parameter. If $P=0$, then equation (4.2) becomes a linear equation. $D_{*}^{\alpha} y(t)=0$,

and when $P=1$, then the equation (4.2) becomes the original equation (1.1). The solution of equation (1.1) can be written as a power series in $P$ as follows: $y(t)=y_{0}(t)+P y_{1}(t)+P^{2} y_{2}(t)+\cdots$

Put $P=1$ in equation (4.4), so the approximate solution of equation (1.1)

$y(t)=y_{0}(t)+y_{1}(t)+y_{2}(t)+\cdots$

Substituting (4.4) in (4.2), and equating the coefficients of like powers of $\mathrm{P}$, we have the following series of linear equations:

$$
\begin{aligned}
& P^{0}: D^{\alpha} y_{0}(t)=0 \\
& \begin{aligned}
P^{1}: D^{\alpha} y_{1}(t)= & f(t)+P(t) y_{0}(t) \\
& \left.\quad+\int_{0}^{t} k(t, s) y_{0}(s) d s\right), \\
P^{2}: D^{\alpha} y_{2}(x)= & P(t) y_{1}(t) \\
& \left.\quad+\int_{0}^{t} k(t, s) y_{1}(s) d s\right), \\
P^{3}: D^{\alpha} y_{3}(x)= & P(t) y_{2}(t) \\
& \left.+\int_{0}^{t} k(t, s) y_{2}(s) d s\right),
\end{aligned}
\end{aligned}
$$

It is obvious that these equations can be easily solved by applying the operator $I^{\alpha}$, the inverse of the operator $D^{\alpha}$, which is defined by (2.2). Hence, the components $y_{0}(t), y_{1}(t), y_{2}(t)+\cdots$ of the HPM solution can be determined. That is, in (4.5) we can determine the HPM series solutions.

\section{NUMERICAL EXAMPLES}

In this section, we have applied Legendre wavelet method and He's homotopy perturbation method for linear fractional Integro-differential equations with known exact solution. All the results are calculated by using the symbolic computation software Maple 16.

Example 5.1. Consider the following fractional integrodifferential equation:

$$
\begin{aligned}
& D_{*}^{0.75} y(t) \\
& =\frac{t^{0.25}}{\Gamma(1.25)}-t-t^{2}-\frac{1}{2} t^{3}-\frac{1}{3} t^{4}+t y(t) \\
& +\int_{0}^{t} t s y(s) d s
\end{aligned}
$$

with the initial condition

$y(0)=1$,

with the exact solution

$y(t)=1+t$.

According to LWM, with $K=2$ and $M=2$. We have $f(t)=$ $\frac{t^{0.25}}{\Gamma(1.25)}-t-t^{2}-\frac{1}{2} t^{3}-\frac{1}{3} t^{4}, \quad P(t)=t, k(t, s)=t s, \quad \alpha=$ $0.75, m=0,1, \quad L=1, \quad n=1,2$, $\psi_{n, m}(t)$

$= \begin{cases}\sqrt{m+\frac{1}{2}} 2^{\frac{k}{2}} P_{m}(4 t-\hat{n}), & \frac{\hat{n}-1}{4} \leq t<\frac{\hat{n}+1}{4}, \\ 0 & \text { otherwise }\end{cases}$

We get a system of equations from

$\sum_{n=1}^{2} \sum_{m=0}^{1} \sum_{r=0}^{1} \omega_{r}\left(\psi_{n, m}\left(x_{i}-x_{i} r\right)-\psi_{n, m}(0)\right) C_{n m}$

$=\frac{x_{i}^{0.25}}{\Gamma(1.25)}-x_{i}-x_{i}{ }^{2}-\frac{1}{2} x_{i}{ }^{3}-\frac{1}{3} x_{i}{ }^{4}$

$+\sum_{n=1}^{2} \sum_{m=0}^{1} C_{n m} x_{i} \psi_{n, m}\left(x_{i}\right)$

$+\sum_{n=1}^{2} \sum_{m=0}^{1} C_{n m} \int_{0}^{x_{i}} x_{i} s \psi_{n, m}(s) d s$

Where

$x_{i}=\frac{s_{i}+1}{2}$, where $s_{i}=\cos \left(\frac{\pi i}{3}\right), \quad i=1,2,3$,

and from (3.5) we get

$0.75(0.25) \frac{\Gamma(-0.75)}{x_{i}^{-0.75}} \omega_{r}= \begin{cases}-1, & \text { if } r=0, \\ 0.75, & \text { if } r=1 .\end{cases}$

$Y_{2,2}(0)=\sum_{n=1}^{2} \sum_{m=0}^{1} C_{n m} \psi_{n, m}(0)=1$.

Combine equations (5.5) and (5.6) to obtain 4 linear equations and solve it to get from which

$C_{10}=0.88388, C_{11}=0.10206$,

$C_{20}=1.23740, \quad C_{21}=0.10209$.

Then the approximate solution of (5.1) will be $\sum_{n=1}^{2} \sum_{m=0}^{1} C_{n m} \psi_{n, m}(t)$

$= \begin{cases}0.88388(\sqrt{2})+0.10206(\sqrt{6})(4 t-1), & 0 \leq t<0.5, \\ 1.2374(\sqrt{2})+0.10209(\sqrt{6})(4 t-3), & 0.5 \leq t \leq 1,\end{cases}$ 
$= \begin{cases}1.0000+0.99998 t, & 0 \leq t<0.5, \\ 0.99989+1.0003 t, & 0.5 \leq t \leq 1 .\end{cases}$

We compute the absolute error $E_{1}=\left|y_{A P P}-y_{\text {exact }}\right|$, where $y_{\text {exact }}=1+t$, and $y_{A P P}$ is the approximate solution of (5.1) by using LWM that given by (5.7), (see Table 1).

According to HPM we construct the following homotopy

$$
\begin{gathered}
D_{*}^{0.75} y(t)=P\left(\frac{t^{0.25}}{\Gamma(1.25)}-t-t^{2}-\frac{1}{2} t^{3}-\frac{1}{3} t^{4}+t y(t)\right. \\
\left.\quad+\int_{0}^{t} t s y(s) d s\right)
\end{gathered}
$$

substituting (4.4) in (5.8), we obtain the following series of equations with identical power of $P$ :

$P^{0}: D^{0.75} y_{0}(t)=0$,

$$
\begin{aligned}
P^{1}: D^{0.75} y_{1}(t)= & \frac{t^{0.25}}{\Gamma(1.25)}-t-t^{2}-\frac{1}{2} t^{3}-\frac{1}{3} t^{4}+t y_{0}(t) \\
& +\int_{0}^{t} t s y_{0}(s) d s,
\end{aligned}
$$

$P^{2}: D^{0.75} y_{2}(x)=t y_{1}(t)+\int_{0}^{t} t s y_{1}(s) d s$

$P^{3}: D^{0.75} y_{3}(x)=t y_{2}(t)+\int_{0}^{t} t s y_{2}(s) d s$,

Consequently, by applying the operator $I^{0.75}$ to the above sets we get:

$$
\begin{aligned}
& y_{0}(t)=1 \text {, } \\
& y_{1}(t)=t-0.4521829620 t^{2.75}-0.3046285217 t^{4.75} \text {, } \\
& y_{2}(t)=0.4521829620 t^{2.75}-0.1432862435 t^{4.5} \\
& -0.09679336446 t^{6.5} \\
& +0.1015428406 t^{4.75} \\
& -0.008965361090 t^{8.5} \text {, }
\end{aligned}
$$

Therefore the approximate solution of (5.1) will be

$$
\mathrm{y}(\mathrm{t})=\mathrm{y}_{0}(\mathrm{t})+\mathrm{y}_{1}(\mathrm{t})+\mathrm{y}_{2}(\mathrm{t})+\cdots
$$$$
=1+\mathrm{t} \text {. }
$$

We compute the absolute error $E_{2}=\left|y_{A P P}-y_{\text {exact }}\right|$, where $y_{\text {exact }}=1+t$, and $y_{A P P}$ is the approximate solution of (5.1) by using HPM that given by (5.9), (see Table 1). Comparison between the approximate solutions obtained by LWM and HPM and the exact solution, (see Fig.1).

Table 1. The absolute errors of example 5.1.

\begin{tabular}{|c|c|c|}
\hline $\mathrm{t}$ & Abs. $E_{1}(\mathrm{LWM})$ & Abs. $E_{2}(\mathrm{HPM})$ \\
\hline 0.0 & 0.0 & 0.0 \\
\hline 0.1 & $2.0 \mathrm{E}-6$ & 0.0 \\
\hline 0.2 & $4.0 \mathrm{E}-6$ & 0.0 \\
\hline 0.3 & $6.0 \mathrm{E}-6$ & 0.0 \\
\hline 0.4 & $8.0 \mathrm{E}-6$ & 0.0 \\
\hline 0.5 & $4.0 \mathrm{E}-5$ & 0.0 \\
\hline 0.6 & $7.0 \mathrm{E}-5$ & 0.0 \\
\hline
\end{tabular}

\begin{tabular}{|c|c|c|}
\hline 0.7 & $1.0 \mathrm{E}-4$ & 0.0 \\
\hline 0.8 & $1.3 \mathrm{E}-4$ & 0.0 \\
\hline 0.9 & $1.6 \mathrm{E}-4$ & 0.0 \\
\hline 1.0 & $1.9 \mathrm{E}-4$ & 0.0 \\
\hline
\end{tabular}

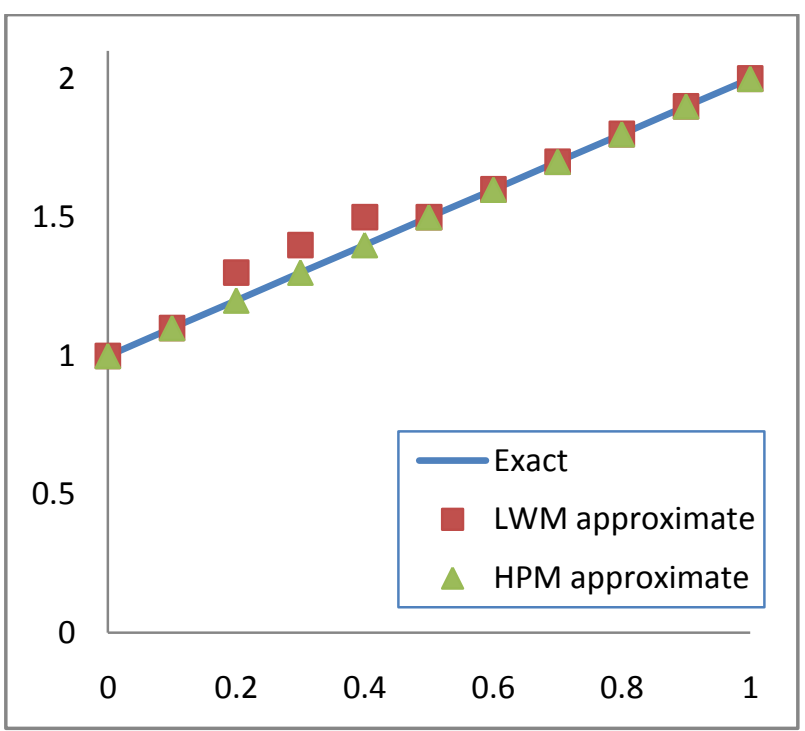

Fig.1. comparison of approximate solutions obtained by LWM and HPM with the exact solution of example 5.1.

Example 5.2. Consider the following fractional integrodifferential equation:

$$
\begin{gathered}
D_{*}^{0.25} y(t)=\frac{2 t^{1.75}}{\Gamma(2.75)}+e^{t}\left(1+\frac{1}{2} t^{2}-\frac{1}{3} t^{3}\right)-e^{t} y(t) \\
+\int_{0}^{t} e^{t} s y(s) d s,
\end{gathered}
$$

with the initial condition

$y(0)=1$,

with the exact solution

$y(t)=1+t^{2}$

According to LWM we solved the linear system that was obtained by equations (3.2) and (3.6) with $K=2$ and $M=2$ to get:

$C_{10}=0.7535243273, \quad C_{11}=0.0267948984$,

$C_{20}=1.0953173580, \quad C_{21}=0.1226796548$.

Then the approximate solution of (5.10) will be

$\sum_{n=1}^{2} \sum_{m=0}^{1} C_{n m} \psi_{n, m}(t)$

$= \begin{cases}1.000000000+0.2625364145 t, & 0 \leq t<0.5 \\ 0.6474863647+1.202015258 t, & 0.5 \leq t \leq 1 .\end{cases}$

We compute the absolute error $E_{3}=\left|y_{A P P}-y_{\text {exact }}\right|$, where $y_{\text {exact }}=1+t^{2}$, and $y_{A P P}$ is the approximate solution of (5.10) by using LWM that given by (5.13), (see Table 2).

According to HPM we construct the following homotopy 


$$
\begin{gathered}
D_{*}^{0.25} y(t)=P\left(\frac{2 t^{1.75}}{\Gamma(2.75)}+e^{t}\left(1+\frac{1}{2} t^{2}-\frac{1}{3} t^{3}\right)-e^{t} y(t)\right. \\
\left.+\int_{0}^{t} e^{t} s y(s) d s\right)
\end{gathered}
$$

substituting (4.4) in (5.14), we obtain the following series of equations with identical power of $P$ :

$P^{0}: D^{0.75} y_{0}(t)=0$,

$$
\begin{aligned}
P^{1}: D^{0.25} y_{1}(t)= & \frac{2 t^{1.75}}{\Gamma(2.75)}+e^{t}\left(1+\frac{1}{2} t^{2}-\frac{1}{3} t^{3}\right)-e^{t} y_{0}(t) \\
& +\int_{0}^{t} e^{t} s y_{0}(s) d s
\end{aligned}
$$

$P^{2}: D^{0.25} y_{2}(x)=-e^{t} y_{1}(t)+\int_{0}^{t} e^{t} s y_{1}(s) d s$,

$P^{3}: D^{0.25} y_{3}(x)=-e^{t} y_{2}(t)+\int_{0}^{t} e^{t} s y_{2}(s) d s$

By applying the operator $I^{0.25}$ to the above sets and to avoid the difficult fractional integral, we can simplify the integrations by taking the truncated Taylor expansions for the exponential term: $\left(e^{t} \cong 1+\frac{t}{1 !}+\frac{t^{2}}{2 !}+\frac{t^{3}}{3 !}\right)$ to get the following

$$
y_{0}(t)=1 \text {, }
$$$$
y_{1}(t)=0.9999999998 t^{2}+0.7845423297 \cdot t^{2.25}
$$

$$
\begin{aligned}
& +0.4827952799 t^{3.25} \\
& +0.1135988894 t^{4.25} \\
& +0.03462061391 t^{6.25}
\end{aligned}
$$

$y_{2}(t)=I^{0.25}\left[-e^{t} y_{1}(t)+\int_{0}^{t} e^{t} s y_{1}(s) d s\right]$,

Therefore the approximate solution of (5.10) will be

$$
\begin{aligned}
\mathrm{y}(\mathrm{t}) & =\mathrm{y}_{0}(\mathrm{t})+\mathrm{y}_{1}(\mathrm{t})+\mathrm{y}_{2}(\mathrm{t})+\cdots \\
& =1+0.9999999998 t^{2} .
\end{aligned}
$$

We compute the absolute error $E_{4}=\left|y_{A P P}-y_{\text {exact }}\right|$, where $y_{\text {exact }}=1+t^{2}$ and $y_{A P P}$ is the approximate solution of (5.10) by using HPM that given by (5.15), (see Table 2).

Comparison between the approximate solutions obtained by LWM and HPM and the exact solution, (see Fig.2).

Table 2. The absolute errors of example 5.2.

\begin{tabular}{|c|c|c|}
\hline $\mathrm{t}$ & Abs. $E_{3}(\mathrm{LWM})$ & Abs. $E_{4}(\mathrm{HPM})$ \\
\hline 0.0 & 0.0 & 0.0 \\
\hline 0.1 & $1.625364145 \mathrm{E}-2$ & $2.0 \mathrm{E}-12$ \\
\hline 0.2 & $1.250728290 \mathrm{E}-2$ & $8.0 \mathrm{E}-12$ \\
\hline 0.3 & $1.123907565 \mathrm{E}-2$ & $1.8 \mathrm{E}-11$ \\
\hline 0.4 & $5.49854342 \mathrm{E}-2$ & $3.2 \mathrm{E}-11$ \\
\hline 0.5 & $1.50600630 \mathrm{E}-3$ & $5.0 \mathrm{E}-11$ \\
\hline 0.6 & $8.69551950 \mathrm{E}-3$ & $7.2 \mathrm{E}-11$ \\
\hline
\end{tabular}

\begin{tabular}{|l|l|l|}
\hline 0.7 & $1.10295470 \mathrm{E}-3$ & $9.8 \mathrm{E}-11$ \\
\hline 0.8 & $3.09014289 \mathrm{E}-2$ & $1.2 \mathrm{E}-10$ \\
\hline 0.9 & $8.06999033 \mathrm{E}-2$ & $1.62 \mathrm{E}-10$ \\
\hline 1.0 & $1.50498377 \mathrm{E}-1$ & $2.0 \mathrm{E}-10$ \\
\hline
\end{tabular}

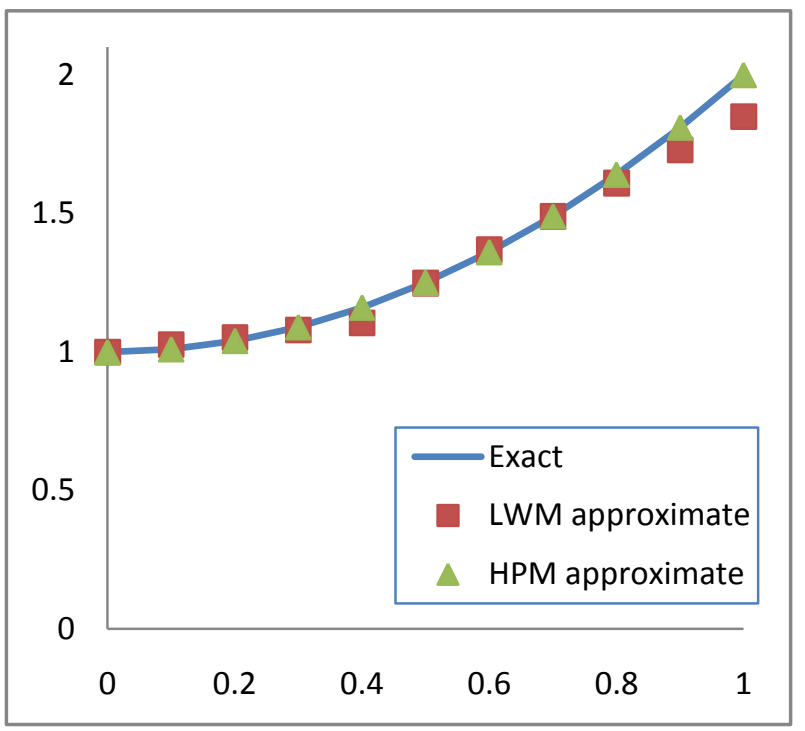

Fig.2. comparison of approximate solutions obtained by LWM and HPM with the exact solution of example 5.2.

Example 5.3. Consider the following fractional integrodifferential equation:

$D_{*}^{0.5} y(t)$

$$
\begin{aligned}
& =\frac{2 t^{0.5}}{\Gamma(1.5)}+\sin t+t(2 t \cos t+\cos t-1)-\sin t y(t) \\
& +\int_{0}^{t} t \sin s y(s) d s
\end{aligned}
$$

with the initial condition

$y(0)=1$,

with the exact solution

$y(t)=1+2 t$.

According to LWM we solved the linear system that was obtained by equations (3.2) and (3.6) with $K=2$ and $M=2$ to get:

$$
\begin{aligned}
& C_{10}=1.06066, \quad C_{11}=0.20412, \\
& C_{20}=1.76780, \quad C_{21}=0.20414 .
\end{aligned}
$$

Then the approximate solution of (5.15) will be

$$
\begin{aligned}
& \sum_{n=1}^{2} \sum_{m=0}^{1} C_{n m} \psi_{n, m}(t) \\
& = \begin{cases}1.0000+2.0000 t, & 0 \leq t<0.5, \\
0.9999+2.0002 t, & 0.5 \leq t \leq 1 .\end{cases}
\end{aligned}
$$

We compute the absolute error $E_{5}=\left|y_{A P P}-y_{\text {exact }}\right|$, where $y_{\text {exact }}=2 t+1$, and $y_{A P P}$ is the approximate solution of (5.15) by using LWM that given by (5.18), (see Table 3 ).

According to HPM we construct the following homotopy 


$$
\begin{aligned}
& D_{*}^{0.5} y(t) \\
& =P\left(\frac{2 t^{0.5}}{\Gamma(1.5)}+\sin t+t(2 t \cos t+\cos t-1)-\sin t y(t)\right. \\
& \left.+\int_{0}^{t} t \sin s y(s) d s\right)
\end{aligned}
$$

Substituting (4.4) in (5.19), we obtain the following series of equations with identical power of $P$ :

$$
\begin{aligned}
& P^{0}: D^{0.5} y_{0}(t)= 0, \\
& P^{1}: D^{0.5} y_{1}(t)= \frac{2 t^{0.5}}{\Gamma(1.5)}+\sin t+t(2 t \cos t+\cos t-1) \\
& \quad-\sin t y_{0}(t)+\int_{0}^{t} t \sin s y_{0}(s) d s, \\
& P^{2}: D^{0.5} y_{2}(x)=-\sin t y_{1}(t)+\int_{0}^{t} t \sin s y_{1}(s) d s, \\
& P^{3}: D^{0.5} y_{3}(x)=-\sin t y_{2}(t)+\int_{0}^{t} t \sin s y_{2}(s) d s,
\end{aligned}
$$

By applying the operator $I^{0.5}$ to the above sets and to avoid the difficult fractional integral, we can simplify the integrations by taking the truncated Taylor expansions for the trigonometric terms:

$$
\left(\cos t \cong 1-\frac{t^{2}}{2 !}+\frac{t^{4}}{4 !}-\frac{t^{6}}{6 !}, \sin t \cong t-\frac{t^{3}}{3 !}+\frac{t^{5}}{5 !}-\frac{t^{7}}{7 !}\right)
$$

to get the following

$y_{0}(t)=1$,

$y_{1}(t)=2.000000000 t+1.203604445 t^{2.5}$

$0.4585159790 t^{4.5}+0.03206405448 t^{6.5}$

$0.0009388690461 t^{8.5}-0.000007941561481 t^{9.5}$,

$y_{2}(t)=I^{0.5}\left[-\sin t y_{1}(t)+\int_{0}^{t} t \sin s y_{1}(s) d s\right]$,

Therefore the approximate solution of (5.10) will be

$$
\mathrm{y}(\mathrm{t})=\mathrm{y}_{0}(\mathrm{t})+\mathrm{y}_{1}(\mathrm{t})+\mathrm{y}_{2}(\mathrm{t})+\cdots
$$$$
=1+2.000000000 t \text {. }
$$

We compute the absolute error $E_{6}=\left|y_{A P P}-y_{\text {exact }}\right|$, where $y_{\text {exact }}=1+2 t$, and $y_{A P P}$ is the approximate solution of (5.15) by using HPM that given by (5.20), (see Table 3 ).

Comparison between the approximate solutions obtained by LWM and HPM and the exact solution, (see Fig.3).

Table 3. The absolute errors of example 5.3.

\begin{tabular}{|c|c|c|}
\hline $\mathrm{t}$ & Abs. $E_{5}(\mathrm{LWM})$ & Abs. $E_{6}(\mathrm{HPM})$ \\
\hline 0.0 & 0 & 0 \\
\hline 0.1 & 0 & 0 \\
\hline 0.2 & 0 & 0 \\
\hline 0.3 & 0 & 0 \\
\hline 0.4 & 0 & 0 \\
\hline
\end{tabular}

\begin{tabular}{|l|c|c|}
\hline 0.5 & 0 & 0 \\
\hline 0.6 & $2 \mathrm{E}-5$ & 0 \\
\hline 0.7 & $4 \mathrm{E}-5$ & 0 \\
\hline 0.8 & $6 \mathrm{E}-5$ & 0 \\
\hline 0.9 & $8 \mathrm{E}-5$ & 0 \\
\hline 1.0 & $10 \mathrm{E}-5$ & 0 \\
\hline
\end{tabular}

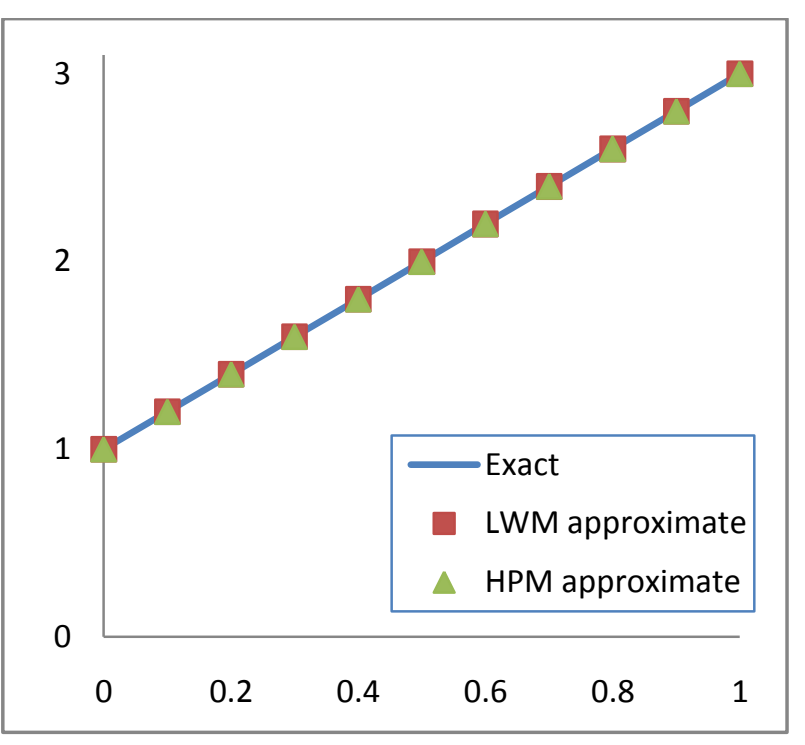

Fig.3. Comparison of approximate solutions obtained by LWM and HPM with the exact solution of example 5.3.

\section{CONCLUSION}

In this paper, this study showed that for most linear fractional integro-differential equations we usually derive very good approximations to the solutions. It can be concluded that the LWM and HPM are a powerful and efficient technique in finding very good solutions for this kind of equations. We find that the results in HPM are better than the results in LWM (see Fig.1, Fig.2 and Fig.3).

\section{ACKNOWLEDGMENTS}

Our thanks to the experts who have contributed towards development of the template.

\section{REFERENCES}

[1] Adam Loverro, Fractional calculus: History, Definitions and Application for the Engineer. (2004).

[2] D. Elliott, An asymptotic analysis of two algorithms for certain Hadamard finitepart integrals, IMA J. Numer. Anal. 13 (1993), 445-462.

[3] E. A. Rawashdeh, Numerical of fractional integrodifferential equations by collocation method, Appl. Math. Comput. 176 (2006) 1-6.

[4] E. A. Rawashdeh, Legendre Wavelet method for fractional integro-differential equations, Applied Mathematics Sciences.5 (2011) 2467-2474.

[5] H. Saeedi, F. Samimi, He's Homotopy perturbation method for nonlinear Ferdholm integro-differential equations of fractional order, Applied International Journal of Engineering Research and Applications.Vol.2 (2012) 2248-9622. 
[6] I. Podlubny, Fractional Differential Equations, Academic press, New York, 1999.

[7] J.-H. He, "Homotopy perturbation method: a new nonlinear analytical technique," Applied Mathematics and Computation, vol. 135, no. 1, pp. 73--79, 2003.

[8] J.-He, "Homotopy perturbation technique," Computer Methods in Applied Mechanics and Engineering, vol. 178, no. 3-4, pp. 257--262, 1999.

[9] J.-H. He, "Homotopy perturbation method with an auxiliary term," Abstract and Applied Analysis, vol. 2012, Article ID 857612, 7 pages, 2012.

[10] K. Diethelm, An algorithm for the numerical solution of differential equations of fractional order, Electronic Trans. Num. Ana. 5 (1997), 1-6.
[11] K. Miller, B. Ross, An Introduction to the Fractional Calculus and Fractional Differential, Willey, New York, 1993.

[12] L. Huang, X. Li, Y. Zhao, X. Duan, Approximate solution of fractional integro-differential equations by Taylor expansion method, Computers and Mathematics with Application, 62 (2011) 1127-1134.

[13] R.C. Mittal, R. Nigam, Solution of fractional integrodifferential equations by Adomian decomposition method, Int. J. of Appl. Math. and Mech, 4 (2) (2008) 87-94.

[14] S. M. Momani, local and global existence theorems integro-differential equations, Jornal of Fractional Calculus, 18 (2000) 81-86.

[15] S.Yousefi and M. Razzaghi, Legendre wavelets method for the nonlinear Volterra-Fredholm integral equations, Mathematics and computers in simulation 70 (2005), 1-8 